5. Ward C, Walters EH, Zheng L, et al. Increased soluble CD14 in bronchoalveolar lavage fluid of stable lung transplant recipients. Eur Respir J 2002;19:472-8.

\section{Repeated thrombolytic therapy after initial unsuccessful thrombolysis in massive pulmonary embolism}

In patients with massive pulmonary embolism threatened by haemodynamic instability, thrombolytic treatment is recommended. ${ }^{1}$ But when it fails, therapeutic options remain limited and are mainly guided by local expertise. In the current case, we report a potential treatment modality for this situation.

A 60-year-old male patient collapsed several times at home. He had a history of a curative left upper lobe lung resection 2 months previously because of a squamous cell lung carcinoma. On admission he suffered from severe dyspnoea. Physical examination showed an elevated central venous pressure and a systolic fixed splitting of the valve sounds. His systolic blood pressure was just below $80 \mathrm{~mm} \mathrm{Hg}$, with a pulse of $122 \mathrm{bpm}$. Thoracic CT angiography (angio-CT) confirmed suspected massive pulmonary embolism (fig 1A) with an increased right ventricular diameter/left ventricular diameter ratio ( $\mathrm{RV} / \mathrm{LV}$ ratio) of 1.76. Because of haemodynamic instability, he received thrombolysis with alteplase (10 $\mathrm{mg}$ bolus, $90 \mathrm{mg} / 2 \mathrm{~h}$ ), which stabilised his systolic blood pressure at around $100 \mathrm{~mm} \mathrm{Hg}$, and then was commenced on nadroparin and coumarins.

Despite thrombolytic treatment, he still had severe orthostatic hypotension, and 5 days after the initial event he collapsed again. Repeat angio-CT showed the same configuration of pulmonary embolism (fig $1 \mathrm{~B}$ ), with an $\mathrm{RV} / \mathrm{LV}$ ratio of 1.57 . We thereafter started treatment with urokinase using an initial bolus infusion of $2000 \mathrm{IU} / \mathrm{kg}$ and continuous infusion at $2000 \mathrm{IU} / \mathrm{kg} / \mathrm{h}$ for $48 \mathrm{~h}$; in the meantime, nadroparin was continued. Within the first $24 \mathrm{~h}$, the patient's clinical condition did not improve but at $48 \mathrm{~h}$ he had neither symptoms of orthostatic hypotension nor resting tachycardia. Angio-CT after $48 \mathrm{~h}$ of urokinase treatment showed no signs of residual central embolism (fig 1C) and a decrease in $\mathrm{RV} / \mathrm{LV}$ ratio to 1.12 , indirectly indicating a decrease in right ventricular overload. Four days later the patient was discharged.

To date, apart from a very small $(n=8)$ randomised trial, there is no solid scientific evidence for using thrombolytic agents in the treatment of patients with haemodynamic instability due to massive pulmonary embolism. ${ }^{2}$ This worsens when treatment fails in this situation, which has been reported to occur in up to $8 \%$ of patients. ${ }^{3}$ When initial thrombolytic treatment fails, surgical rescue embolectomy, interventional radiology or using a second thrombolytic drug remain treatment options. ${ }^{1}$ Recently, a single centre registry showed that rescue embolectomy resulted in lower inhospital mortality compared with treatment with a second thrombolytic drug. ${ }^{3}$ In this study, the second attempt to achieve thrombolysis was performed with either streptokinase or alteplase, depending on which drug had been given previously, but only for a $2 \mathrm{~h}$ period. Based on this evidence, the alternative treatment option in our case would have been surgical pulmonary embolectomy which, in skilled hands, has a 1 year survival
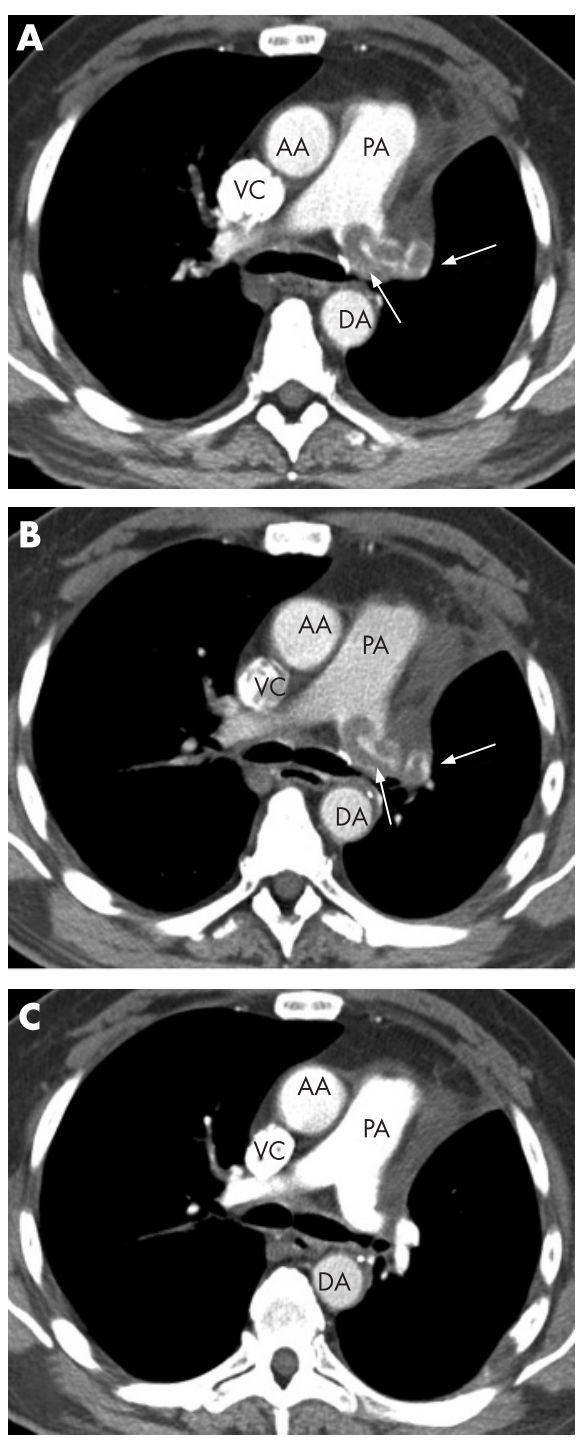

Figure 1 (A-C) Selected pictures from CT angiography (angio-CT) at the main pulmonary artery (PA) level. (A) On admission. (B) Five days after alteplase treatment. (C) Forty-eight hours after initiation of urokinase treatment. Note: the patient had a lobectomy of the left upper lobe (see text). AA, ascending aorta; $D A$, descending aorta; $V C$, superior caval vein. The white arrows indicate the actual pulmonary embolism at this section of the angio-CT. rate of $86 \% .^{3}$ However, because of the patient's recent thoracic surgery as well as the availability of an alternative treatment option, we decided not to perform surgery.

In our case, we choose to give urokinase for a prolonged period, considering the short half life of thrombolytic agents (alteplase 4$6 \mathrm{~min}$, urokinase 4-20 min, streptokinase 18-23 $\mathrm{min}$ ) as well as the fact that alteplase in a $2 \mathrm{~h}$ regimen might be too short to achieve lysis of an extensive clot. Although the effectiveness of prolonged alteplase (24 $72 \mathrm{~h}$ ) for venous thromboembolic disease has been reported, we choose a different agent because of its reported initial ineffectiveness in our patient. ${ }^{5}$ In addition, our preference for urokinase over alteplase was related to our previous experiences with this regimen and its known capacity to induce thrombolysis in longstanding clots.

Prolonged thrombolytic treatment in patients with massive pulmonary embolism, who fail to respond to initial alteplase therapy, might be considered a good treatment alternative.

\section{R J M van den Biggelaar, ${ }^{1}$ D-J Slebos, ${ }^{1} \mathrm{~J}$ van der} Meer $^{2}$

'Department of Pulmonary Diseases, Thrombosis and Rheology, University Medical Centre Groningen, Groningen, The Netherlands; ${ }^{2}$ Division of Haemostasis, Thrombosis and Rheology, University Medical Centre Groningen, Groningen, The Netherlands

Correspondence to: Dr D-J Slebos, Department of Pulmonary diseases, University Medical Centre Groningen, PO Box 30001, 9700 RB Groningen, The Netherlands; d.j.slebos@int.umcg.n

Competing interests: None.

Thorax 2008:63:89. doi:10.1136/thx.2007.084616

\section{REFERENCES}

1. Kucher N, Goldhaber SZ. Management of massive pulmonary embolism. Circulation 2005:112:e28-32.

2. Jerjes-Sanchez C, Ramirez-Rivera A, de Lourdes García M, et al. Streptokinase and heparin versus heparin alone in massive pulmonary embolism: a randomized controlled trial. J Thromb Thrombolysis 1995:2:227-9

3. Meneveau N, Seronde MF, Blonde MC, et al. Management of unsuccessful thrombolysis in acute massive pulmonary embolism. Chest 2006;129:1043-50.

4. Leacche M, Unic D, Goldhaber SZ, et al. Modern surgical treatment of massive pulmonary embolism: results in 47 consecutive patients after rapid diagnosis and aggressive surgical approach. J Thorac Cardiovasc Surg 2005:129:1018-23.

5. Protack CD, Bakken AM, Patel N, et al. Long-term outcomes of catheter directed thrombolysis for lower extremity deep venous thrombosis without prophylactic inferior vena cava filter placement. J Vasc Surg 2007:45:992-7.

6. Pilloud J, Rimensberger PC, Humbert J, et al. Successful local low-dose urokinase treatment of acquired thrombosis early after cardiothoracic surgery. Pediatr Crit Care Med 2002:3:355-7.

\section{Bronchial hyperresponsiveness (BHR) and physical activity}

We read with interest the recent paper by Shaaban et al ${ }^{1}$ who report a negative 